\title{
DIE ENTE - DER HEILIGE VOGEL DER GOTEN?
}

\author{
A N D R ZEJ KOKOWSKI ${ }^{\circledR}$ - TERESA M A Z U REK
}

\begin{abstract}
The Duck - the Sacred Bird of the Goths? With three new finds of the Masłomęcz type duck fibulae, their number has increased to 14. The concentration of these primers in the Masłomęcz group and the surrounding area seems to confirm the thesis about their local production. In the essay, the duck is presented as an ornamental motif in the cultures of the Gothic circle of the Late Roman Period, which indicates that this bird played a certain role in the beliefs of the Goths.
\end{abstract}

Keywords: Eastern Poland, Roman Period, Goths, Sarmatians, religion, bird fibula.

Im Jahre 1980 wurde in Masłomęcz, Gem. Hrubieszów, Woi. Lubelskie, Fpl. 15 ein interessantes Körpergrab Nr. 10 entdeckt (Kokowski 1983, Taf. 302). Mit dieser Entdeckung kamen zwei wichtige Kulturmerkmale zum Vorschein, die später als einige der Ausgliederungsgrundlagen der gotischen Masłomęcz-Gruppe gedient haben (Kokowski 1997; 2009, 187-198). Erstens, die im Grab bestattete Frau war ohne Kopf beigesetzt, was auf die Sitte der Zerstückelung der Leiche vor dem Begräbnis hinweist. Das zweite Merkmal ist eine Armbrustfibel A VII mit dem Bügel in Form einer schwimmenden Ente. Die effektvolle Fibel wurde bald ein Zeichen, sozusagen ein Wappen nicht nur der MasłomęczGruppe, aber auch der Lubliner Archäologie (Kokowski 2007a, 36, 37; 2012, 76-79; 2014, 78-81).

Im Gräberfeld Masłomęcz wurden noch drei weitere Entenfibeln entdeckt. Kindergrab 56 enthielt zwei solche Exemplare (Kokowski/Koman 1985, Taf. 337: 1, 2), das dritte kam in Grab 460 zum Vorschein (Kokowski 2003, 278, Abb. 1: d). Somit wurde eine These gestellt, dass die Entenfibeln hochwahrscheinlich als lokale Herstellung der MasłomęczGruppe gelten sollen. Sie datieren in die Stufe Cla.

Einen Anlass zum vorgelegten Aufsatz sind drei neue Funde der Entenfibeln und die Notwendigkeit, den vierten ähnlichen Fund von Grabowiec voll zu publizieren, der bisher nur aus einem Foto bekannt ist (Kokowski 2007b, Einkleber; s. auch Bender 2009, 56, Abb. 24: 3), der zuerst als römisch, nicht lokal interpretiert wurde. Alle Exemplare sind aus Bronze hergestellt.

1. Buśno, Gem. Białopole (AZP 83-92), Kr. Chełm, Woi. lubelskie. Einzelfund, ohne genaue Lokalisation. Bügel gerade, dachförmig, unten abgeflacht, L. $30 \mathrm{~mm}$. Fuß schwalbenschwanzförmig, Br. $10 \mathrm{~mm}$, mit von unten befestigtem halbrundem Halter zum Achsenloch, Achse nicht erhalten. Oberteil des Bügels verdickt und verbreitert, mit stilisiertem „Entenkopf“" auf kurzem, romboidalem, $3 \mathrm{~mm}$ hohem „Hals" beendet. Auf dem „Entenkopf ${ }^{\text {" }}$ feine, schräge Kerben. Unter dem Bügel nicht ganz erhaltener Nadelhalter, erhaltene H. 8 mm, Br. 10 mm. Rohstoff: Kupferlegierung (nach metallographischer Analyse im Labor der Staatlichen Beruflichen Hochschule in Chełm), $\mathrm{Sn}-38,977 \%$; $\mathrm{Cu}-24,069 \%$; $\mathrm{Pb}-16,092 \%$; $\mathrm{Fe}-7,432 \%$; $\mathrm{Si}-6,588 \%$; $\mathrm{Ca}-5,797 \%$; $\mathrm{Ag}-0,355 \%$; $\mathrm{Sb}-0,355 \%$; $\mathrm{Zn}-$ 0,177 \%; Sc - 0,108 \%; Zr - 0,035 \%. Sammlung: Muzeum Ziemi Chełmskiej in Chełm, Inv.-Nr.: MZCH/A/9925; Kat.-Nr. 7687 (Abb. 1: 3; 2: 1).

2. Ukraine (Westukraine?), Fundort unbekannt. Aus dem Internetforum der "Schatzsucher" Mit dem Fund von Buśno fast identisch. Von allen Fibeln des Masłomęcz-Typs unterscheidet sich die Fibel daran, dass statt eines Fußes mit Schwanz ist ein hinter der Mutter schwimmendes Kücken-Entlein dargestellt. Ausmaße unbekannt (Abb. 1: 1).

3. Ostpolen (vermutlich vom Bereich der MasłomęczGruppe oder Umgebung), Fundort unbekannt. Aus dem Internetforum der "Schatzsucher". Wie der Fund von Buśno, jedoch unverziert, Schwanz fächerförmig. Ausmaße unbekannt (Abb. 1: 2).

4. Grabowiec, Gem. loco, Kr. Zamość, Woi. lubelskie. Ohne genaue Lokalisation, Sammlung der prähistorischen und frühmittelalterlichen Funde aus der Umgebung von Grabowiec (Kokowscy 2012, 252). Bronzene Armbrustfibel, Bügel gebogen, im Querschnitt romboidal, in Form einer schwimmenden Ente. Niedriger Nadelhalter unter dem Hals des stilisierten Vogels. Kurzer Schwanz, schräg gekerbt. L. 48 mm, Br. 3-6 mm, H. 1 mm, Spirallänge $16 \mathrm{~mm}$. Stilistisch wie Entenfibeln A VII von der Masłomęcz-Gruppe. Privatbesitz (Abb. 1: 4; 2: 2).

Ein gemeinsames Merkmal der Fibeln vom Masłomęcz-Typ ist, dass die Achsen des Bügels,

\footnotetext{
1 Die Fibeln Nr. 3 und 4 wurden zwar auf dem karierten Papier fotografiert, doch ein kariertes Feld schwankt zwischen $4 \times$ $4 \mathrm{~mm}$ und $6 \times 6 \mathrm{~mm}$. Die Größe der Fibel lässt sich somit nicht ganz genau bestimmen.
} 


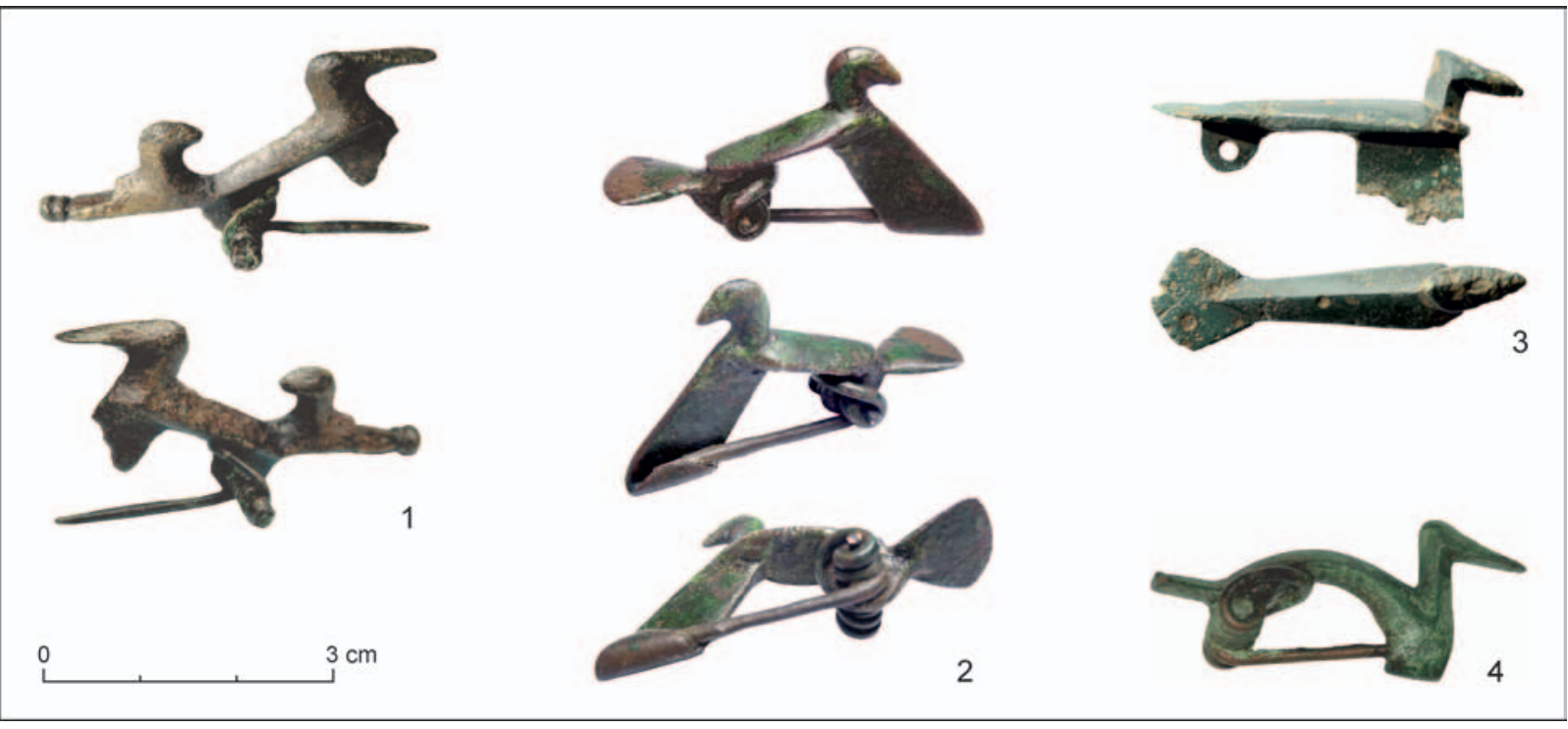

Abb. 1. Entenfibeln. 1 - „Westukraine“; 2 - „Ostpolen“; 3 - Buśno; 4 - Grabowiec.
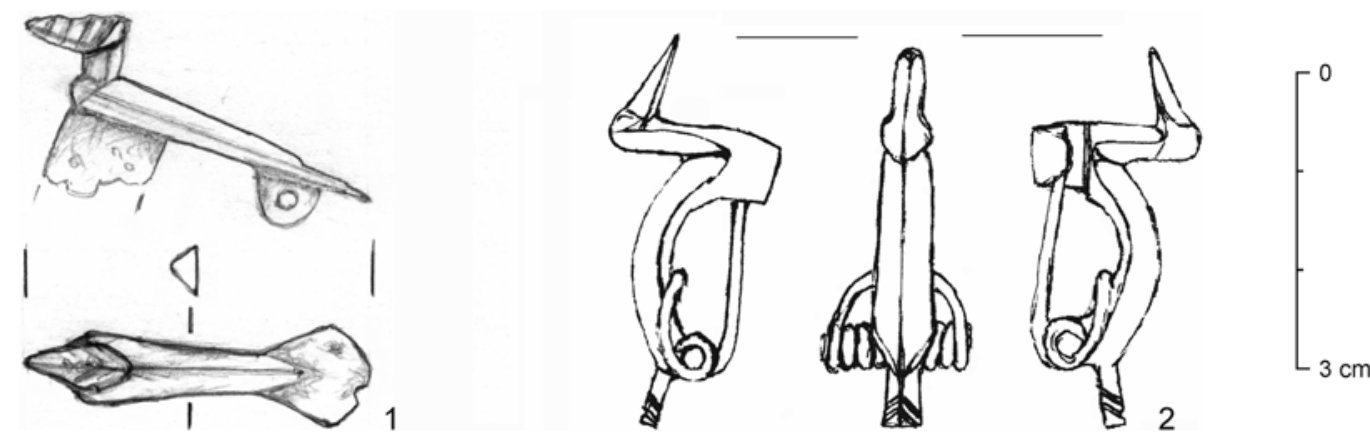

Abb. 2. Entenfibeln. 1 - Buśno; 2 - Grabowiec (Zeichnung T. Mazurek).

des hohen, meistens leicht nach unten verbreiterten Nadelhalters und der Nadel ein etwa rechtwinkliges Dreieck bilden. Darüber, auf der längeren Seite, ist eine von unten flacher Darstellung einer schwimmenden Ente angebracht. Der unprofilierte, trapezförmige Fuß bildet die Verlängerung der Bügelachse. Alle Fibeln sind etwa gleich groß, dagegen ist ihre Verzierung differenziert, wie Augen oder ihr Mangel, verschiedene Kerben auf dem Schnabel und fuß; der letztgenannte manchmal mit Zahnornament, das Feder markiert.

Das Exemplar von Grabowiec steht stilistisch den oben beschriebenen nahe, jedoch ist der Nadelhalter niedrig, der Bügel in Form des Entenkörpers gebogen und der parallel zur rechteckigen, nicht trapezförmigen, im Querschnitt dachförmigen Verschlussachse verlaufende Fuß. Der Fund findet seine fast genaue Entsprechung in der Fibel vom Kultplatz der Przeworsk-Kultur in Otalążka, Kr. Radom (Skorupka 2008, 42, Abb. 26) und in der Fibel von Pritzier, Grab 846, Ldkr. Ludwigslust-Parchim (Schuldt 1955, 69, Abb. 328) in Mecklenburg-Vorpommern (vgl. Bender 2008, 56-60). Auf die römische Herkunft des Exemplars von Otalążka weist die metallographische Analyse hin, die eine komplizierte, „nichtbarbarische“ Rezeptur aufweist (Biborski 2009).

Im Barbaricum haben wir also mit zwei unterschiedlichen Mustern der Entenfibeln, Masłomęcz und Otalążka zu tun. Zur dritten Gruppe gehören die Vogelfibeln mit dem Bügel in Form einer Taube, diese aber sind vor allem aus den römischen Provinzen bekannt.

Die neueste Studie über die kaiserzeitlichen Entenfibeln im mitteleuropäischen Barbaricum 


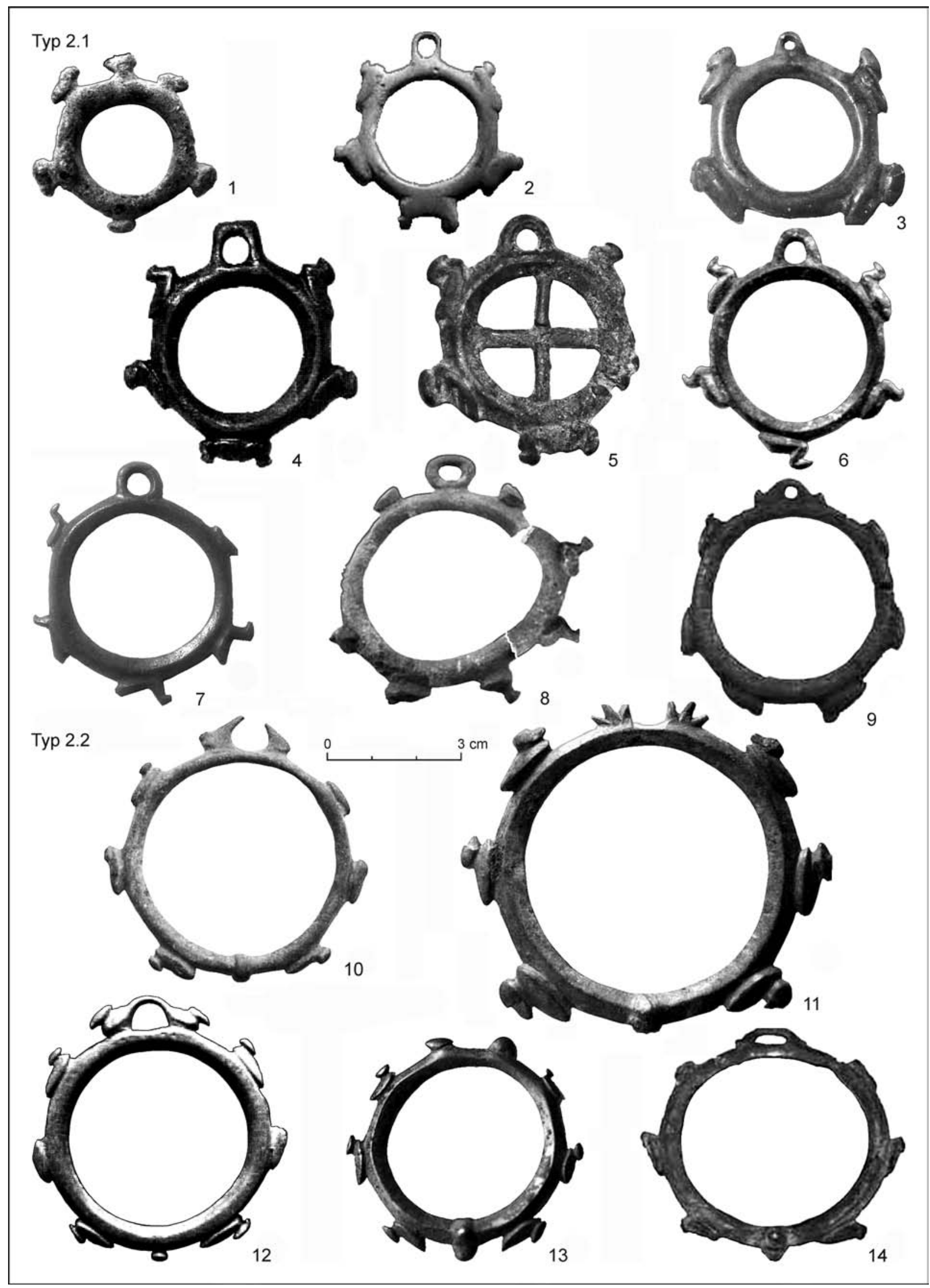

Abb. 3. Entenringe der Černjachov-Kultur (nach Magomedov 2018). 
verdanken wir E. Droberjar (2018). Er hat elft ihm bekannte Exemplare von sieben im römischen Sinn „barbarischen“ Fundstellen zusammengestellt und ihre Verwandtschaft mit provinzialrömischen Fibeln hervorgehoben: vier von Masłomęcz, zwei von Ostróżek Wielki (eigentlich Wielki Ostróżek [Великий Острожок], Rai. Chmel'nyc'kyj, obl. Vinnycja, Ukraine)2; je ein von Piatrovičy (Пятровічы, Weißrussland; Beljavec 2016, 411, Abb. 8); Csongrád - Sövényháza; Martfü, Zsófia-Meierei (beide Ungarn - s. unten); Vrbová Lhota (Böhmen) und ein stark im Feuer deformiertes Exemplar von Ulów, Fpl. 7, Gem. und Kr. Tomaszów Lubelski (Niezabitowska-Wiśniewska 2015, 334, 335, Abb. 2: 1). Die Funde von Otalążka und Pritzier blieben dagegen von ihm unberücksichtigt. E. Droberjar hat auch auf die Verwandtschaft der Masłomęcz-Fibeln mit der Gruppe der römischen Vogelfibeln aufmerksam gemacht, unter denen die Taubenfibeln überwiegen (vgl: Steinklauber 2010, 21, 22, Foto 2: 3; Taf. 2: 2, 3). Unter ihnen befinden sich auch solche, die als Entenfibeln bezeichnet werden können, wie das von E. Droberjar angeführte Exemplar von Letcombe Regis (Oxford) auf den Britischen Inseln. Es ist jedoch zu bemerken, dass die Unterschiede zwischen Bezeichnungen Taube/Ente oft intuitiv sind. Am Rand unserer Erwägungen ist es zu erwähnen, dass bisher aus dem Barbaricum nur zwei römische Taubenfibeln stammen, eine vom unbekannten Fundort in der Ukraine, vielleicht aus der Gegend von Odessa (Sammlung: Odesskij Gorodskij Archeologičeskij Muzej, Nr. OGAM, A-4425: Ambroz 1966, 36, Taf. 21; hier als "sitzende Taube " beschrieben) und eine vom ebenfalls unbekannten Fundort in der Slowakei (Sammlung: damaliges Slowakisches Museum in Bratislava, heute Slovenské národné múzeum Archeologické múzeum; Lamiová-Schmiedlová 1961, 131, Nr. 256; Taf. XI: 10).

Wie schwierig ist, einen Fund als barbarisch bzw. antik zu bestimmen, sehen wir am folgenden Beispiel: die silberne Entenfibel vom Typ Masłomęcz aus dem sarmatischer Grab in Martfü, ZsófiaMeierei, hielt E. Droberjar für barbarisch, während nach $A$. Vaday (2005, 77, Taf. 4: 7) wurde sie als provinzialrömisch betrachtet. Die Fibel unterscheidet sich von jenen vom Typ Masłomęcz nicht nur vom Rohstoff, aber auch davon, dass der Vogelkörper verziert ist (was bisher bei den Masłomęcz-Fibeln nicht vorkommt). Darüber hinaus ist der Nadelhalter bei dem Exemplar von Martfü durchlocht und mit einer Kette versehen. An den Achsenenden hängen an Ketten zwei halbmondförmige Anhänger, schließlich ist der Schwanz ebenfalls durchlocht, woran vermutlich derselbe Anhänger ebenfalls hing. Dies aber kann eine lokale, sarmatische Adaptation des germanischen Musters sein, die dem lokalen Geschmack entsprach. Keinem Zweifel unterliegt dagegen der zweite Fund aus dem sarmatischen Bereich, von Csongrád-Sövényháza, da die dort gefundene Bronzefibel sicher nach dem Masłomęcz-Muster hergestellt wurde (Párducz 1950, 155, Taf. XXVII: 1).

Das Vorkommen der Entenfibel vom Typ Masłomęcz im sarmatischen Bereich wurde ein unter anderen Argumenten für die These für die Kontakte der Goten mit den sarmatischen Nomaden bereits in der mittleren Kaiserzeit (B2/C1-C1a; Kokowski 2003; 2004; 2019, 59-61).

Die Bevölkerung des gotischen Kulturkreises ${ }^{3}$ verwendete figürliche Motive äußerst selten, wobei am häufigsten die stilisierte Entendarstellung auftritt, besonders auf den in der Černjachov-Kultur verbreiteten kreisförmigen Anhängern mit Entenminiaturen am Umfang (Abb. 3; Magomedov 2018). Das Entenmotiv kommt auch auf der silbervergoldeten Pressblechapplikation mit Gesichtsmaske mit Delphinen und Räubervogelköpfen von Grab 453 in Masłomęcz vor (Abb. 4; Kokowski 2001, 39, Abb. 4). Sie kann aber skandinavischer Herkunft sein, da in Skandinavien die vergoldeten Pressblecharbeiten am häufigsten auftreten (vgl. von Carnap-Bornheim/ Ilkjær 1996, 409-470).

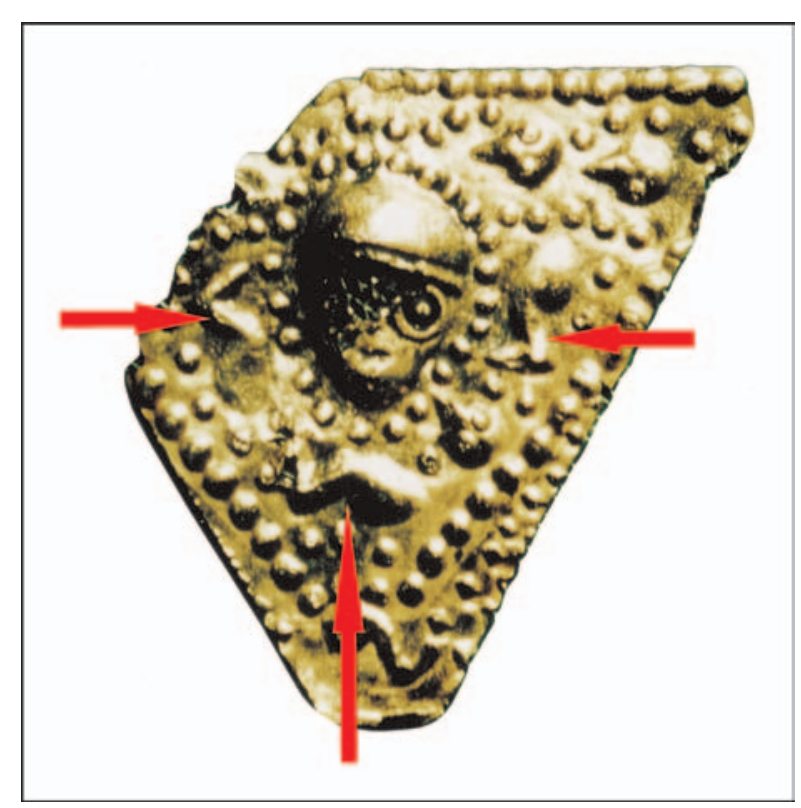

Abb. 4. Pressblech mit Gesichtsmaske von Grab 453 in Masłomęcz. Die Entendarstellungen sind mit Pfeilen markiert. Ohne Maßstab (Foto A. Kokowski).

\footnotetext{
2 Die beste Dokumentation dieser Funde s. Gałęzowska 2010, 251-254, Abb. 2: 1; 3.

3 Dazu zählen die Wielbark-, Černjachov-, Sântana de Mureş-Kulturen und die Masłomęcz-Gruppe (Kokowski 1997).
} 


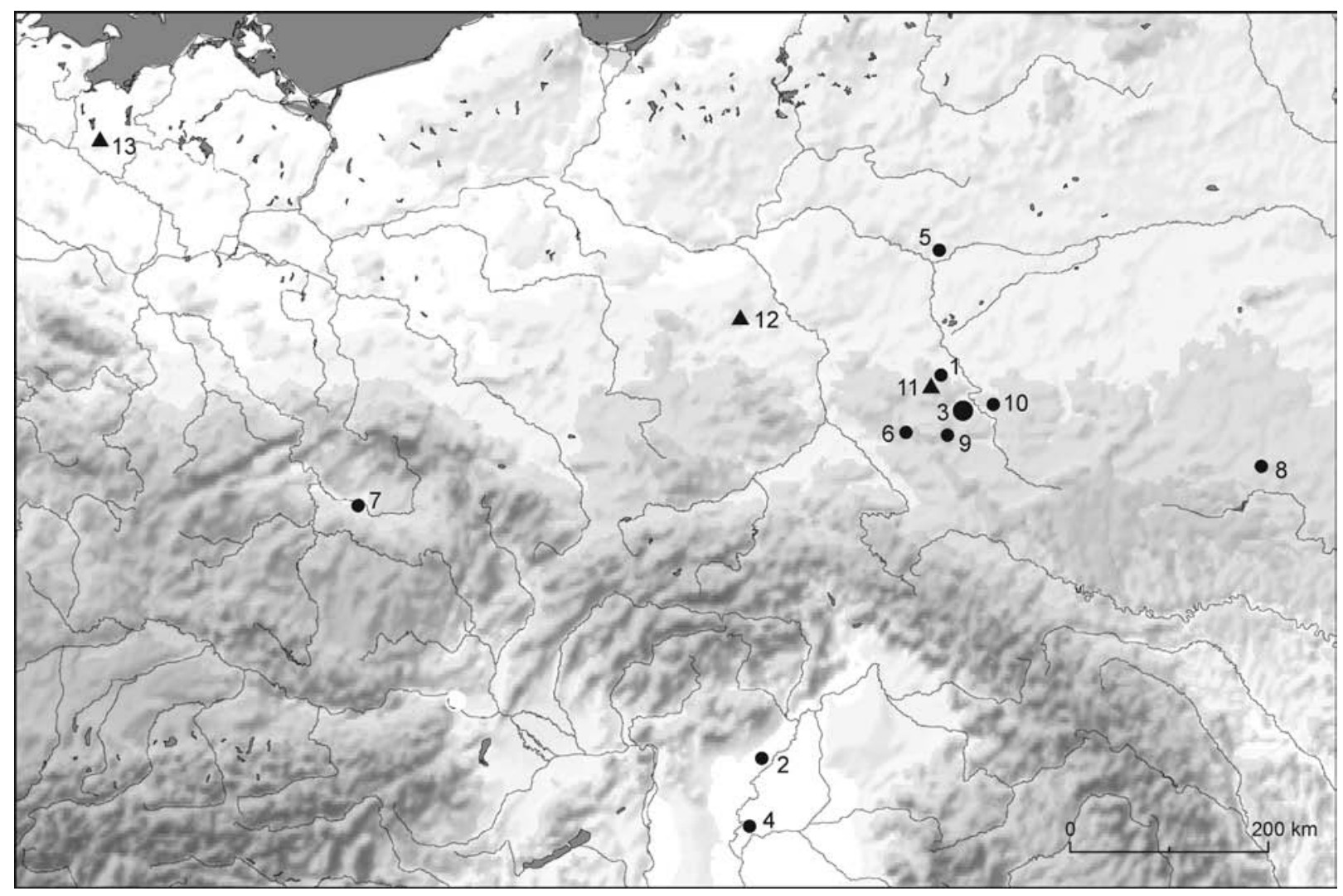

Abb. 5. Verbreitung der Vogelfibeln in Barbaricum. Kreis - Masłomęcz-Muster: 1 - Buśno; 2 - Csongrád-Sövényháza; 3 - Masłomęcz, Fpl. 15; 4 - Martfü; 5 - Piatrovičy; 6 - Ulów, Fpl. 7; 7 - Vrbová Lhota; 8 - Wielki Ostróżek; 9 - „Ostpolen“; 10 - „Westukraine“. Dreieck - Otalążka-Muster: 11 - Grabowiec; 12 - Otalążka; 13 - Pritzier.

Viele Archäologen sehen fast in jeder figürlichen Darstellung eine symbolische Bedeutung aus dem Glaubens- und Religionskreis. Die Vögel sind dabei überall vertreten (Skorupka 2008, 35-41); die Ente ist von vielen, antiken und barbarischen Glaubenssystemen bekannt (s. Lunczer 2009, 48; Rowland 1978, 49-51). Daher tritt sie vermutlich auch in der provinzialrömischen Welt auf (s. Patek 1942, 295; Riha 1979, 199; Vaday 1981). Wir verfügen zwar über keine Angaben zur wahrsagerischen Rolle der Enten, es gibt jedoch noch ein Argument für ihre wichtige Rolle in der Glaubenswelt.

In der Masłomęcz-Gruppe herrschte die Sitte, den Verstorbenen ins Grab Tiere beizulegen. Meistens sind es einzelne Stücke, seltener einige, wie Säugetiere, Amphibien, Fische und Vögel (Rogatko
1991). Unter den letztgenannten befinden sich auch Enten (Kalisz 2006).

Wahrscheinlich ist es etwas übertrieben, die Enten als ",heilige Vögel“ bei den Goten zu betrachten, doch kann ihre Symbolik in der kaiserzeitlichen geistigen Kultur eine wichtige Rolle gespielt haben. Die Anzahl der Entenfibeln vom Typ Masłomęcz heute 14 Exemplare - in der Masłomęcz-Gruppe und in ihrer Nachbarschaft scheint die These über ihre lokale Herstellung zu belegen (Abb. 5). Es fehlen leider präzise Argumente für ihr Auftreten in Mittelböhmen und bei den sarmatischen Jazygen. Hier müssen wir uns mit einer allgemein angenommenen These über die Kulturkontake zwischen Hrubieszów-Becken und genannten Gebieten befriedigen.

\section{LITERATUR}

Ambroz 1966 - A. K. Ambroz: Fibuly juga evropejskoj časti SSSR II v. do n. e. $-I V$ v. n. e. Archeologija SSSR. Svod archeologičeskich istočnikov D1-30. Moskva 1966.

Beljavec 2016 - V. G. Beljavec: Nasel'nictva vel'barskaj kul'tury ŭ historyka-kul'turnym razvicci zjamel'
Belarusi rubjažu II/III - pačatku V st. n. è. In: O. N. Levko/D. V. Duk (red.): Slavjane na territorii Belarusi v dogosudarstvennyj period. Kniga I. K 90-letiju so dnja roždenija doktora istoričeskich nauk, profesora Leonida Davydoviča Pobolja. Minsk 2016, 384-450. 
Bender 2009 - W. Bender: Otalażka. Stanowisko kultowo-bagienne z młodszego okresu rzymskiego. Materiały Starożytne i Wczesnośredniowieczne 8. Warszawa 2009.

Biborski 2009 - M. Biborski: Wyniki badań składu chemicznego zapinki z Otalążki, pow. grójecki, stan. 1. In: Bender 2009, 93-96.

von Carnap-Bornheim/Ilkjær 1996 - C. von Carnap-Bornheim/J. Ilkjær: Illerup Ådal 5-7. Die Prachtausrüstungen. Jutland Archaeological Society Publications 25. Arrhus - Moesgård 1996.

Droberjar 2018 - E. Droberjar: Eine Vogelfibel mit hohem Nadelhalter vom Typ Masłomęcz aus Vrbová Lhota (Mittelböhmen). In: B. NiezabitowskaWiśniewska/P. Łuczkiewicz/S. Sadowski/M. StasiakCyran/M. Erdrich (red.): Studia Barbarica. Profesorowi Andrzejowi Kokowskiemu w 65. rocznice urodzin. Tom II. Lublin 2018, 74-84.

Gałęzowska 2010 - A. Gałęzowska: Znaleziska z okresu rzymskiego z terenu Ukrainy w zbiorach Muzeum Archeologicznego w Poznaniu. In: A. Urbaniak/ R. Prochowicz (red.): Terra Barbarica. Studia ofiarowane Magdalenie Mączyńskiej w 65. rocznicę urodzin. Monumenta Archaeologica Barbarica. Series Gemina 2. Łódź - Warszawa 2010, 251-260.

Kalisz 2006 - J. Kalisz: Pochówki zwierzęce na cmentarzyskach grupy masłomęckiej. In: L. Kostuch/K. Ryszewska (red.): Zwierze jako sacrum w pradziejach $i$ starożytności I. Kielce 2006, 53-59.

Kokowscy 2012 - E. und A. Kokowscy: Kościana rękojeść nahajki z okolic Grabowca, w pow. hrubieszowskim. Wiadomości Archeologiczne 62, 2012, 252-254.

Kokowski 1983 - A. Kokowski: Période Romaine Tardive. Civilisation de Černjachov. Inventaria Archaeologica Pologne 50. Warszawa 1983.

Kokowski 1997 - A. Kokowski: Die Masłomęcz-Gruppe. Ihre Chronologie und Beziehungen innerhalb des gotischen Kulturkreises - ein Beispiel für den kulturellen Wandel der Goten im Verlauf ihrer Wanderungen. Bericht der Römisch-Germanischen Kommission 78, 1997, 641-833.

Kokowski 2001 - A. Kokowski: Romerske glasbægre i Barbaricum H. J. Eggers type 189 i Mellem- og Nordeuropa. Zur Distribution der Glassgefässe Typ Eggers 189 im Gebiet des mittel und nordeuropäischen Barbaricums. Aarbøger for Nordisk Oldkyndighed og Historie 2001, 35-46.

Kokowski 2003 - A. Kokowski: Geschichte der Verbindungen der Goten mit den Sarmaten im Lichte der Untersuchungen der Masłomęcz-Gruppe und der Kulturen des Goten-Kreises. In: C. von Carnap-Bornheim (Hrsg.): Kontakt-Kooperation-Konflikt. Germanen und Sarmaten zwischen dem 1. und dem 4. Jahrhundert nach Christus. Neumünster 2003, 275-302.

Kokowski 2004 - A. Kokowski: Przyczynek do historii kontaktów Germanów i Sarmatów w świetle badań archeologicznych. In: A. Kokowski (red.): Sarmaci $i$ Germanie. Studia Sarmatica 1. Lublin 2004, 11-70.

Kokowski 2007a - A. Kokowski: 30 powodów do dumy z mieszkania w krainie Gotów. Zamość 2007.

Kokowski 2007b - A. Kokowski: Goci-od Skandzy do Campi Gothorum (od Skandynawii do Pótwyspu Iberyjskiego). Warszawa 2007.
Kokowski 2009 - A. Kokowski: Kontinuität und Diskontinuität der Besiedlung in der jüngeren vorrömischen Eisenzeit und in der römischen Kaiserzeit am Beispiel des Hrubieszów-Beckens. Přehled výzkumů 50, 2009, $181-212$

Kokowski 2012 - A. Kokowski: Gocka duma Masłomęcza. Masłomęcz - Hrubieszów - Lublin 2012.

Kokowski 2014 - A. Kokowski: Gocka duma Masłomęcza. Archeologiczne dziedzictwo regionu hrubieszowskiego. Masłomęcz - Hrubieszów - Lublin 2014.

Kokowski 2019 - A. Kokowski: Illerup Ådal 15. Kleinfunde von zivilem Charakter. Jutland Archaeological Society Publications 25. Århus - Moesgård 2019.

Kokowski/Koman 1985 - A. Kokowski/W. Koman: Néolithique et la période romaine aux environs de Hrubieszów, Pologne de l'est. Inventaria Archaeologica Pologne 54. Warszawa 1985.

Lamiová-Schmiedlová 1961 - M. Lamiová-Schmiedlová: Spony z doby rímskej na Slovensku. Študijné zvesti AÚ SAV 5, 1961, 7-142.

Lunczer 2009 - C. Lunczer: Vögel in der griechischen Antike. Eine Untersuchung über Kenntnisse und Wahrnehmung der antiken Vogelwelt. Disertation. Ruprecht-KarlsUniversität Heidelberg. Philosophishen Fakultät. Schorndorf 2009. Online verfügbar unter: http:// archiv.ub.uni-heidelberg.de/volltextserver/10154/1/ Lunczer_Diss_Voegel.pdf

Magomedov 2018 - B. Magomedov: Ring-Shaped Pendants „with Ducks" of Chernyakhov Culture. In: B. Niezabitowska-Wiśniewska/P. Łuczkiewicz/S. Sadowski/ M. Stasiak-Cyran/M. Erdrich (red.): Studia Barbarica. Profesorowi Andrzejowi Kokowskiemu w 65. rocznice urodzin - For profesor Andrzej Kokowski on his $65^{\text {th }}$ Birthday. Tom I. Lublin 2018, 502-511.

Niezabitowska-Wiśniewska 2015 - B. Niezabitowska-Wiśniewska: Ulów, stanowisko 7 - nowe cmentarzysko kultury wielbarskiej na Roztoczu Środkowym - z badań nad kontaktami kultury wielbarskiej i kultury czerniachowskiej. In: M. Fudziński/H. Paner (red.): Kontakty ponadregionalne kultury wielbarskiej. Przemiany kulturowe w okresie wptywów rzymskich na Pomorzu. Gdańsk 2015, 331-362.

Párducz 1950 - M. Párducz: A Szarmatakor emlékei Magyarorsyágon III. Archaeologia Hungarica 30. Budapest 1950.

Patek 1942 - E. Patek: A Pannóniai fibulatípusok elterjedése és eredete - Verbreitung und Herkunft der römischen Fibeltypen in Pannonien. Dissertationes Pannonicae. Ser. II. Fasc. 19. Budapest 1942.

Riha 1979 - E. Riha: Die römischen Fibeln aus Augst und Kaiseraugst. Forschungen in Augst 3. Augst 1979.

Rogatko 1991 - J. Rogatko: Znaleziska szczątków zwierzęcych na cmentarzyskach grupy masłomęckiej i kultury czerniachowskiej. Archeologia Polski 36, 1991, 151-191.

Rowland 1978 - B. Rowland: Birds with Human Souls. A Guide to Bird Symbolism. Knoxville 1978.

Schuldt 1955 - E. Schuldt: Pritzier. Ein Urnenfriedhof der späten römischen Kaiserzeit in Mecklenburg. Deutschen Akademie der Wissenschaften zu Berlin. Schriften der Sektion für Vor- und Frühgeschichte 4. Berlin 1955. 
Skorupka 2008 - T. Skorupka: Na skrzydłach przeszłości-On wings of the past. Motywy ptaków na zabytkach archeologicznych z ziem polskich (XI w. p.n.e.-XVII w. n.e.) - The motives of the Birds in the archaeological relicts in the Polish lands (11 ${ }^{\text {th }}$ vent. BC-17 th AC). Katalog wystawy Exhibition Catalogue. Poznań 2008.

Steinklauber 2010 - U. Steinklauber: Der Burgberg von Eppenstein als archäologischer Fundort der Römerzeit

Manuskript angenommen am 15. 7. 2021

Übersetzt von Magdalena Mączyńska

prof. dr hab. Andrzej Kokowski

Instytut Archeologii

Uniwersytet Marii Curie-Skłodowskiej

pl. Marii Curie-Skłodowskiej 4

PL - 20-031 Lublin

berig1@gazeta.pl und der Spätantike. Zeitschrift des Historischen Vereines für Steiermark 101, 2010, 9-34.

Vaday 1981 - A. Vaday: Szarmata sírok Martfü-Zsófia Majorban. Szolnok Megyei Múzeumi Évkönyv, 1981, 5-9.

Vaday 2005 - A. Vaday: Corpus der römischen Funde im europäischen Barbaricum. Ungarn 1. Komitat Szolnok. Budapest 2005. mgr Teresa Mazurek

Muzeum Ziemi Chełmskiej im. W. Ambroziewicza Lubelska 55

PL - 22-100 Chełm

teresamazurek42@gmail.com 
